\title{
Urban Air Mobility Research at the DLR German Aerospace Center - Getting the HorizonUAM Project Started
}

\author{
Bianca I. Schuchardt ${ }^{1}$, Dennis Becker ${ }^{2}$, Richard-Gregor Becker ${ }^{3}$, Albert \\ End $^{4}$, Thomas Gerz' ${ }^{5}$ Frank Meller ${ }^{6}$, Isabel C. Metz', Malte Niklaß ${ }^{7}$, \\ Henry Pak ${ }^{8}$, Prajwal Shiva Prakasha', Sebastian Schier-Morgenthal', \\ Karolin Schweiger ${ }^{1}$, Daniel Sülberg ${ }^{10}$, Majed Swaid ${ }^{7}$, Christoph Torens" \\ Chen $\mathrm{Zhu}^{12}$ \\ DLR - German Aerospace Center, Germany
}

\begin{abstract}
Efficiency, safety, feasibility, sustainability and affordability are among the key characteristics of future urban mobility. The project "HorizonUAM - Urban Air Mobility Research at the German Aerospace Center (DLR)" provides first answers to this vision by pooling existing competencies of individual institutes within DLR. HorizonUAM combines research about urban air mobility (UAM) vehicles, the corresponding infrastructure, the operation of UAM services, as well as public acceptance and market development of future urban air transportation. Competencies and current research topics including propulsion technologies, flight system technologies, communication and navigation go along in conjunction with the findings of modern flight guidance and airport technology techniques. The project analyses possible UAM market scenarios up to the year 2050 and assesses economic aspects such as the degree of vehicle utilization or cost-benefit potential via an overall system model. Furthermore, the system design for future air taxis is carried out on the basis of vehicle family concepts, onboard systems, aspects of safety and security as well as the certification of autonomy functions. The analysis of flight guidance concepts and the sequencing of air taxis at vertidromes is another central part of the project. Selected concepts for flight guidance, communication and navigation technology will also be demonstrated with drones in a scaled urban scenario. This paper gives an overview of the topics covered in the HorizonUAM project, running from mid-2020 to mid-2023, as well as an early progress report.
\end{abstract}

\footnotetext{
${ }^{1}$ Researcher, DLR Institute of Flight Guidance, Braunschweig

${ }^{2}$ Researcher, DLR Institute of Communications and Navigation, Oberpfaffenhofen

${ }^{3}$ Researcher, DLR Institute of Combustion Technology, Cologne

${ }^{4}$ Researcher, DLR Institute of Aerospace Medicine, Hamburg

${ }^{5}$ Researcher, DLR Institute of Atmospheric Physics, Oberpfaffenhofen

${ }^{6}$ Head of department, DLR Institute of System Architectures in Aeronautics, Hamburg

${ }^{7}$ Researcher, DLR Air Transportation Systems, Hamburg

${ }^{8}$ Researcher, DLR Institute of Air Transport and Airport Research, Cologne

${ }^{9}$ Researcher, DLR Institute of System Architectures in Aeronautics, Hamburg

${ }^{10}$ Head of test center, DLR Institute of National Experimental Test Center for Unmanned Aircraft Systems, Cochstedt

${ }^{11}$ Researcher, DLR Institute of Flight Systems, Braunschweig

${ }^{12}$ Researcher, DLR Institute of Communications and Navigation, Oberpfaffenhofen
} 


\section{Introduction}

Urban Air Mobility (UAM) is an evolving research topic and has gained attraction not only from researchers but also from potential manufacturers, operators, investors, regulators as well as the general public [1]. Using the urban airspace for passenger transport promises to overcome restrictions that go along with conventional ground-based traffic by making use of the third dimension. Recent advance in electric propulsion technology has led to a multitude of different concepts for electric aircraft capable of Vertical Take-Off and Landing (eVTOL) envisaged for urban usage [2]. Visions on UAM have been published by various research organizations, UAM start-ups as well as traditional aircraft manufacturers [3]-[9].

The German Aerospace Center (DLR) has been playing an active role in multiple previous UAM projects. They cover the investigation of enabling technologies (myCopter ${ }^{13}[10,11]$ ), urban flight guidance concepts (Metropolis $[12,13]$ ), airspace integration (City-ATM [14], CORUS-XUAM ${ }^{14}$ ), cooperative automation of traffic on road, rail, water and in the air (Verkehr 5.0 ${ }^{15}$, Automover ${ }^{16}$ ). Regional passenger transportation (Silent Air Taxi [15]), autonomous cargo transport (ALAADy [16]), contingency management (voloCHRIS ${ }^{17}$, voloSTREAM ${ }^{18}$ ), multicopter drone acoustic $\left(\mathrm{AACID}^{19}\right)$ as well as the design of vehicle concepts for special urban applications (Urban-Rescue ${ }^{20}$ ) are also among the UAM related topics; just to name a few representatives of DLR's UAM research activities.

The recently started project HorizonUAM ${ }^{21}$ (running from mid-2020 to mid-2023) builds on the findings of the above projects conducted by various institutes. Now, the competencies of ten different DLR institutes are combined in a single research project for a collaborative approach to foster UAM research at DLR. The competencies of the involved institutes range from flight guidance, flight systems and air transport to propulsion technologies and atmospheric physics as well as flight medicine. Research on communication and navigation methods is beneficially integrated together with the findings of modern system architecture, airport technology and flight-testing techniques.

This paper gives an overview on the different topics to be covered in the HorizonUAM project and gives reference to already obtained preliminary findings.

\section{Use Cases and UAM System Simulation}

The term Urban Air Mobility refers to aircraft-based passenger services connecting points in metropolitan areas with each other or with suburbs, major transport hubs or other cities. These services are offered by air transport service providers to all citizens who usually do not hold a pilot license. In order to enable the pickup and drop-off of passengers close to the location of demand, Vertical Take-Off and Landing (VTOL) capable vehicles are mandatory and, thus, are the focus of the HorizonUAM project. Several types of air transport services are proposed and described in literature with different particularities and specific demands on the UAM system [5, 17-19]. For research in DLR's HorizonUAM project a set of five use cases is selected in order to cover the broad spectrum of challenges for vehicles, infrastructure, and operations while minimizing the research effort [22].

- The "Intra-City" use case includes air travel where both the origin and destination are within a metropolitan area. Individual transport demand occurs irregularly and at a wide variety of locations, which is a particular challenge for operations. Especially with regard to Germany, transport distances of this use case are assumed to be up to 50 kilometers. To reflect the size of megacities in Asia and North America, a use case "MegaCity" with transport distances up to 100 kilometer is included in the set of use cases, which requires different vehicle designs than in the Intra-City use case.

- The "Airport Shuttle" use case involves feeder services between the airport and selected locations like the city center, the central business district or other points of interest. According to an analysis of Volocopter [23], the vast majority of the airport-to-city-center routes worldwide is below 30 kilometers. The need to also transport luggage and high requirements of the customers with regard to reliability are some of the key challenges of this use case.

\footnotetext{
${ }^{13}$ www.mycopter.eu

${ }_{14}$ https://www.eurocontrol.int/project/corus-Xuam

15 https://www.dlr.de/content/en/articles/digitalisation/digitalisation-project-transport-5-point-0.html

16 https://www.dlr.de/vf/en/desktopdefault.aspx/tabid-2974/1445 read-51313/

17 https://www.bmvi.de/SharedDocs/DE/Artikel/DG/UAV-Projekte/voloCHRIS.html

18 https://cispa.de/en/research/funded-projects-and-collaborations

19 https://www.dlr.de/fl/en/desktopdefault.aspx/tabid-1149/1737_read-72186/

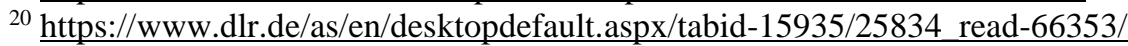

${ }^{21}$ https://www.dlr.de/fl/en/desktopdefault.aspx/tabid-1149/1737_read-69326/
} 
- The "Suburban Commuter" use case represents flights between suburbs and surrounding satellite cities on one side and the city center on the other side which are mainly used by people to commute between home and work place. This use case is challenging in terms of economics because much demand is concentrated in the peak hours, while there is little demand in the off-peak hours and thus the system is underutilized.

- The "Inter-City" use case is characterized by transport of six to ten passengers between cities over distances of more than 100 kilometers. Since VTOL concepts are the focus of the HorizonUAM project, this use case is intended to bridge the gap to aircraft concepts other than VTOL capable vehicles.

For each use case representative missions are defined which in particular form the basis for the vehicle design. A decisive factor here is the number of flight segments that the vehicle can perform without recharging energy. It is envisaged in the Intra-City and Mega-City use case that the vehicles can perform several segments without recharging. This enables the use of simple vertistops without charging possibility which allows the expansion of the network at low cost. For the other use cases, it is foreseen that the vehicle can be recharged immediately after each flight. Furthermore, use case specific characteristics like expected traffic density, structure of the buildup area or customer expectations are relevant aspects for research on flight guidance, communication and navigation, vertidrome technology and operations. Technological progress is represented by two scenarios. The first scenario assumes technologies that will be available in the near future, while the second scenario is based on advanced technologies that will not be available until the middle of this century.

The design of UAM vehicles and the concepts of operation should also consider the influence of meteorological conditions on vehicle and operation and assess the level of noise emission, propagation and immission in the vicinity of vertidromes and along typical flight routes. Vertidromes and vehicles should provide the respective meteorological infrastructure, i.e. sensors and also (access to) local weather forecasts. The project will develop the framework requirements for a safe and noise-propagation reduced UAM traffic in an urban atmospheric boundary layer. Challenges are the channeling and blocking effects in street canyons, resulting in a higher degree of turbulence, and different momentum, heat and energy exchange processes compared to a non-urban environment. The requirements for a high-resolution meteorological sensor technology (able to capture winds with strong gusts and high turbulence levels) on the vehicle and the vertiports will be analyzed. Adequate numerical methods, with a spatial resolution of a few meters and high update rates, to forecast the weather in a heterogeneous and strongly structured environment will be searched and assessed. The forecasts are prerequisite for planning and execution of flights in terms of feasibility and safety and to assess the noise level along flight routes.

In order to assess the UAM system from an economic perspective, the resource planning and optimization are crucial factors. Therefore, an agent-based model is developed. Each agent represents a vehicle with a set of properties such as performance envelope and capacity. Further attributes describe the current vehicle status regarding location, remaining battery charge, the maintenance cycle and several more aspects. Taking these parameters into account, the vehicles are allocated to specific transport requests, such that individual missions can be conducted at a maximum of efficiency and all vehicles in the UAM network are operated at optimum capacity considering least waiting time for passengers, enabling a designed system to operate with a maximum of performance at minimum resource investments. Finally, a methodology for cost evaluation is developed that takes vehicle specific and mission specific effects into account, for instance mission time and energy consumption.

To meet the challenge of modeling and understanding the complex, not yet existing, UAM system, the UAM systems architecture process combines the knowledge of specialists and system engineers and integrates all domain specific analysis components of DLR into an overall UAM system simulation. For connecting the analysis capabilities regarding various disciplines, the Remote Component Environment (RCE) workflow engine is used [24] as it enables the execution of workflows based on "black-boxes" from local and remote locations without jeopardizing intellectual property. In addition to the technical approach of combining disciplinary analysis capabilities, methods of collaboration in teams and knowledge management are mandatory that also integrate experts and their implicit knowledge into the design and simulation process.

The goal of developing a UAM system is to perform comparative analyses of different subsystem designs for the quantification of cause-effect relationships on a system level. In the long term, the UAM system module should enable the derivation of key figures for specific UAM market studies. The individual analysis tools each project partner brings in are utilized within a model based collaborative UAM systems architecture process framework developed from the lessons learnt from collaborative projects at DLR $[25,26]$. The domain capabilities are collected, scenario and use cases defined, CONcepts of OPerations (CONOPs) for vehicles, fleets, and vertiports are defined, Model-Based System Engineering (MBSE) collaborative UAM design is carried out, and finally, sensitivity and tradeoffs are evaluated. The framework enables the project partners to conduct an interdisciplinary and collaborative research to derive and evaluate profitable and sustainable operational concepts in UAM. 
UAM traffic volume is one of the fundamental data necessary to evaluate UAM with regard to feasibility, efficiency, and sustainability. Local authorities and companies are interested in detailed traffic data on the micro level, as e.g. provided by the agent-based models, for the medium- to long-term design and planning of the transportation system. However, estimations of the UAM demand on a national or global scale are also of interest in particular to evaluate the environmental impact of UAM against emission reduction targets and to enable the aviation industry to make the right investment decisions. A number of studies on global UAM demand have already been conducted [2731]. Within the HorizonUAM project agent-based simulations will be set up for selected cities. This will enable improved estimates of the global UAM demand that consider city specific socioeconomic and geographic macro factors as well as supply side characteristics like UAM trip time and costs without the need for elaborate transport modeling.

\section{System-of-System driven Vehicle Design Framework}

Within HorizonUAM a fleet of heterogeneous vehicles is designed in an agent-based simulation embedded systemof-systems (SOS) framework, see Fig. 1. Based on the scenarios, use cases and CONOPS decided at the beginning of the project [22], efficient vehicles are designed to meet the objectives of multiple UAM use cases such as Intra-City, Airport Shuttle, Suburban Commuter, Inter-City and Mega-City. It is envisioned to design multiple vehicle configurations with different passenger capacity, range, technology and speed parameters to be deployed in any given UAM use case. The designed vehicle family is deployed into simulation to evaluate fleet-network efficiency, trajectories, noise and safety. The loop will be iterated till the optimum UAM fleet system-of-systems is evolved. The goal is to establish a family concept with a limited number of individual vehicles. The detailed design work is presented in [32]. The sustainability and life-cycle analysis using system of system approach is also carried out in [33].

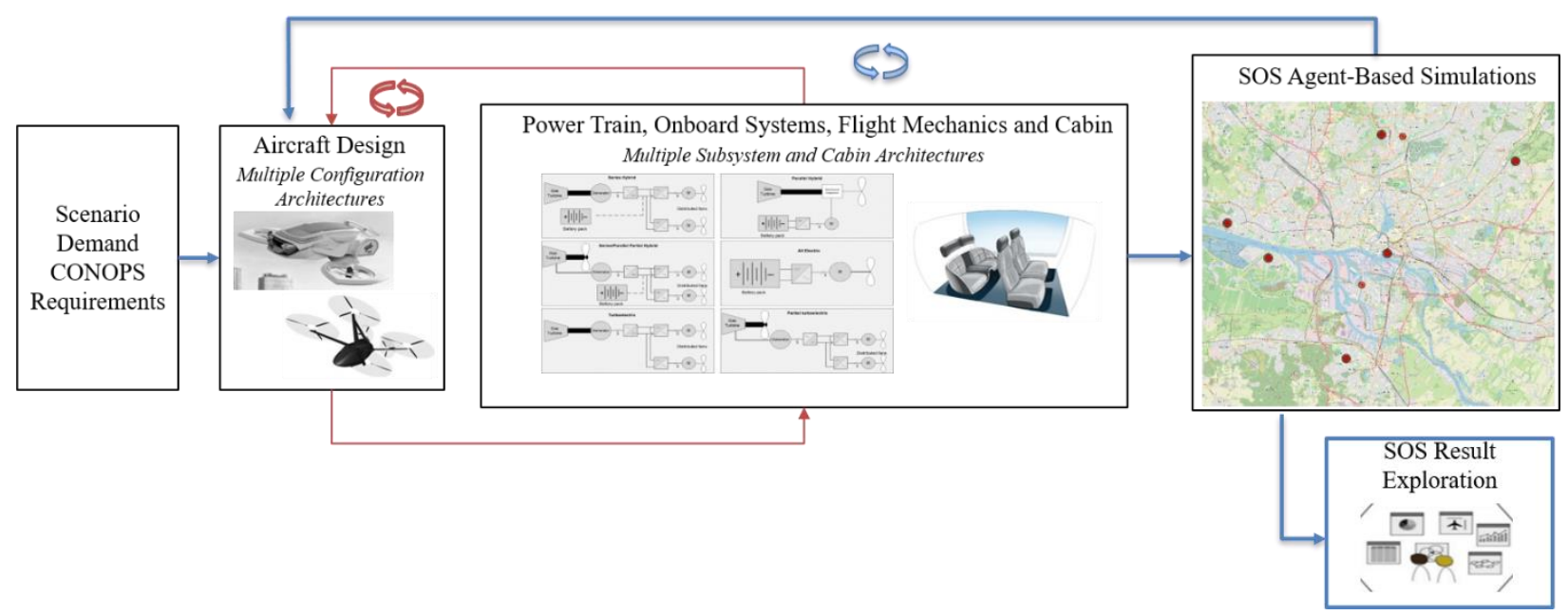

Fig. 1 System-of-systems driven vehicle design framework.

The passenger demand and CONOPS is provided for each use case within the SOS. Apart from configuration architecture specific characteristics such as multirotor or tilt rotor or lift cruise concept etc., overall vehicle and infrastructure technology assumptions are set up for each scenario as shown in Table 1. Here, two different scenarios - near-term and far-term - are considered. In terms of battery energy, note that the battery specific energy is assumed for the pack level. In order to extend battery life, the factor for useable energy is introduced, so that operation at low and high state-of-charge is inhibited. While the near-term assumptions are already optimistic regarding battery technology, the far-term scenario assumes a significantly improved technology level. Several other propulsion concepts are also studied. Furthermore, the UAM operation is expected to be fully autonomous or remote-piloted in the far future, while autonomous deadhead flights (vertidrome reallocation ferry flight) may already be possible in the near term. The requirement for reserve energy is assumed to transition from a time-bound loiter segment into a distance-related diversion segment. Lastly, charging power is not relevant for the sizing process, but will be used in the simulation. Overall, very optimistic and design favoring assumptions are set up in the far-term scenario. It should 
be noted that several factors are sensitive towards vehicle design such as battery composition, energy density, recharge capabilities and weights, power train efficiencies, capacity of the vehicle and the regulation requirement on vehicles such as reserve loiter time. A slight variation of these sensitive parameters will have big impacts on fleet requirements and throughput of the entire UAM system-of-systems.

Table 1 Overall technology assumptions for two different scenarios.

\begin{tabular}{lcc}
\hline Parameter & Near-term scenario & Far-term scenario \\
\hline Battery specific energy, Wh/kg & 300 & 500 \\
Useable energy fraction & 0.80 & 0.90 \\
Sizing mission piloted? & True & False \\
Revenue mission piloted? & True & False \\
Deadhead mission piloted? & False & False \\
Reserve requirement & 20-minute loiter & 5-nmi diversion \\
Charging power, kW & 250 & 1000 \\
\hline
\end{tabular}

With technology assumptions and passenger centric cabin design (Fig. 2), the conceptual vehicle designed is further collaboratively iterated for higher fidelity vehicle design with the project partners (Fig. 3). The collaborative multifidelity approach $[34,35]$ is used for vehicle design considering flight mechanics and stability, detailed offnominal performance constraints, multiple power train and onboard system architecture combinations. Motor battery combinations for vehicle performance needs, weights and power is budgeted for iterated vehicle designs [32].
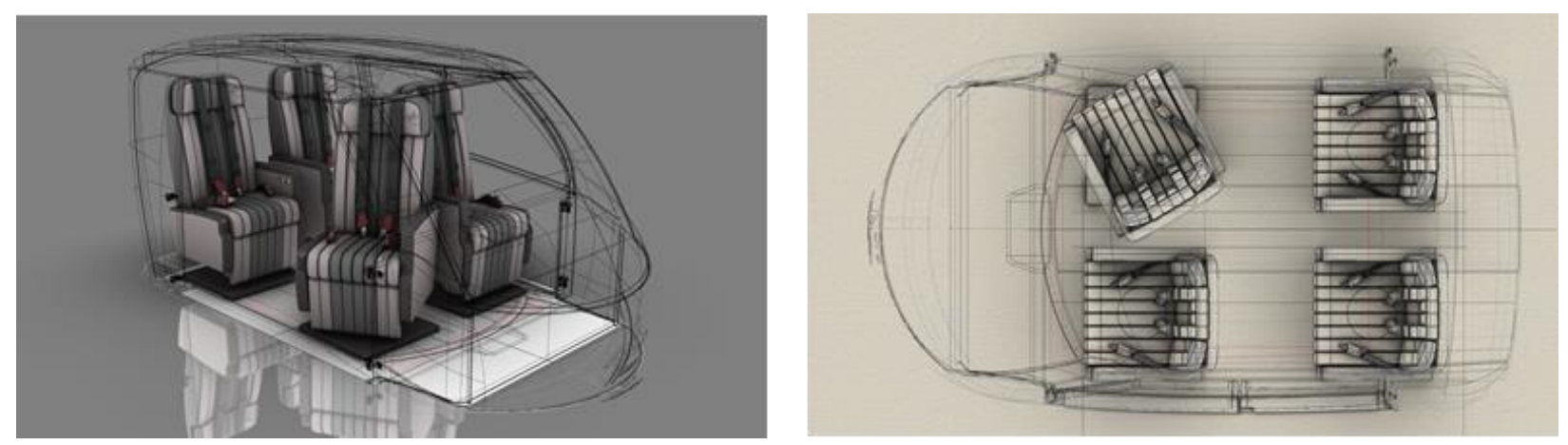

Fig. 2 a) and b) Passenger cabin design architecture, version 1.

The user centric cabin and safety systems are derived with partners for the UAM vehicles via inside out - outside in approach. To understand customer needs, experiences and behavior requirements, persona definition and a passenger acceptance survey were carried out for UAM use cases [36, 37]. Example personas are a 19-years female student, a 35-years male employee, and a 62-years female employee. Each vehicle design is iterated (see Fig. 3) for convergence with optimum cabin, powertrain, subsystem, stability and efficiency. Hence resulting is the optimum heterogeneous fleet for all use cases. 


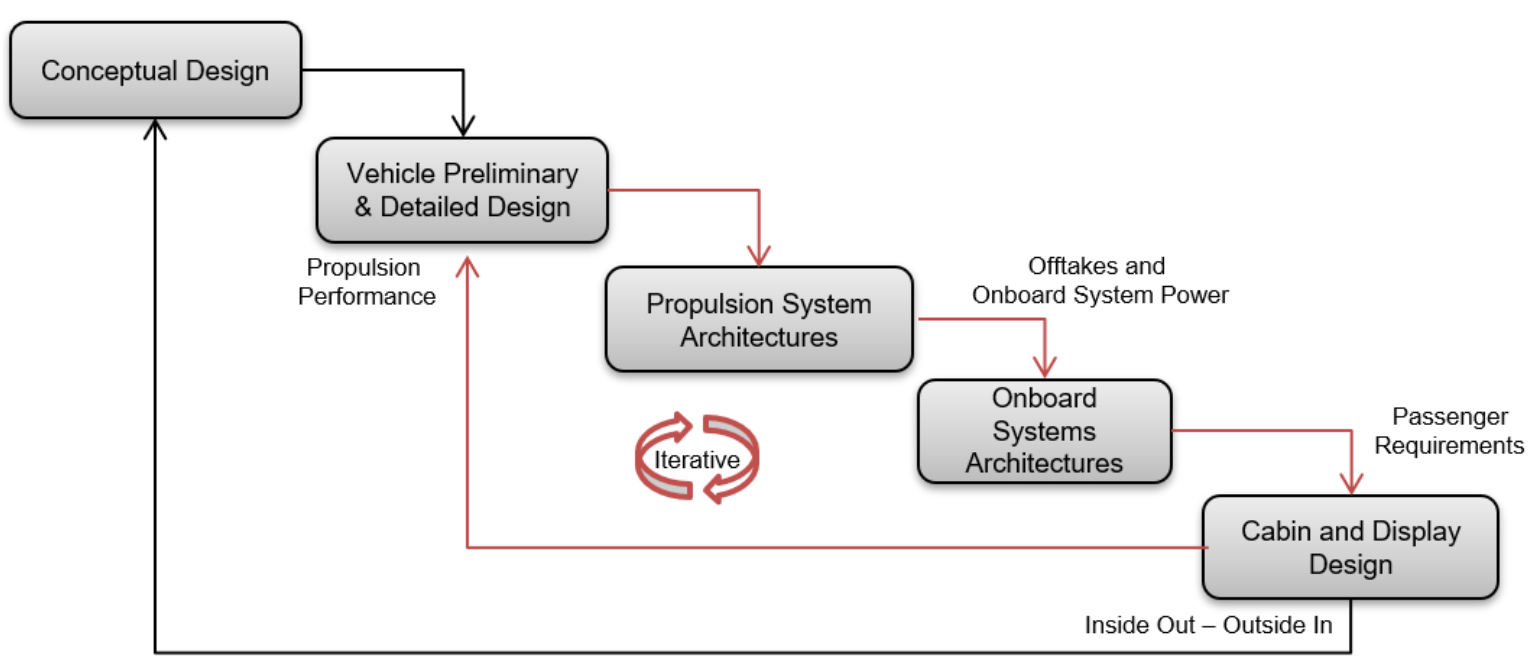

Cost, Energy, Lifecycle and other feedback

Fig. 3 Multifidelity collaborative vehicle design.

\section{Safety and Security}

Large concerns regarding the introduction of urban air mobility are the safety and security aspects for both passengers and people on ground. Upcoming use cases require a high degree of coordination, communication and automation within an urban environment and thus add a lot of complexity to the existing solutions and concepts in aviation. With existing technology, the autonomy of upcoming vehicles and the ability to fly on demand, without a pilot might be an enabler for the scaling and cost effectiveness of business cases for urban air mobility. However, achieving the same levels of safety for urban air mobility will require a lot of effort in research and standardization. It is therefore important to develop autonomous functions in harmony with upcoming regulations and standards. In consequence, the project will research the aspects of safe autonomy. With recent advantages in machine learning, new levels of autonomy are possible for unmanned aviation [38]. These applications are to be extended for increasing the autonomy of UAM vehicles.

Safe, reliable navigation is another key requirement. It is expected that UAM navigation systems are based on redundant sensors and information in order to achieve the required levels of accuracy, integrity, availability and continuity. In order to guarantee safe and smooth operation of the service, the navigation system should ensure sufficient accuracy and simultaneously high reliability, i.e., integrity, of the delivered positioning result. For conventional civil aviation applications, a certification process is required to provide assurances to the safety of the navigation solution. However, the urban operational environment brings new challenges for the navigation system. The vehicles may potentially experience a long period of flight with low satellite visibility and strong environmental multipath effect, especially in deep urban areas. In this situation the performance of Global Navigation Satellite System (GNSS) centered navigation solutions degenerates. As a result, it is necessary to provide a multi-sensor navigation solution including GNSS, kinematic sensors such as IMU (inertial measurement units), and optical sensors such as cameras, etc., in order to cope with the new challenges. Nevertheless, the integrity monitoring of multiple sensor fusion based navigation is still an open topic. Consequently, the certifiability of the solution for safety-critical applications like UAM remains a challenge. We investigate the concept and design of a multi-sensor navigation system that is able to meet the operational UAM safety-related requirements. A system architecture including ground infrastructures and airborne equipment will be considered.

In order to avoid midair collisions, a reliable and trustworthy exchange of position and trajectory information is required. Therefore, it is a project goal to develop a communication concept that enables a safe and secure information exchange in urban airspace. The concept needs to consider the underlying physical propagation characteristics of an urban communication channel in order to address the future requirements of this scenario. First results on drone-todrone communication in urban environments have been published in [39].

With a large number of strongly interconnected, networked autonomous vehicles, cybersecurity threats are even more pressing than with piloted vehicles. In order to recognize such threats and define counter measures at an early stage of the design process, the overall system must be analyzed regarding its structure and interfaces. Therefore, the 
combination of a secure and encrypted data link between the vehicles as well as to air traffic management together with an onboard multisensory navigation system is investigated.

The integration of high numbers of additional airspace users such as drones or air taxis into an airspace management system is essential for a scalable UAM system. The term UTM (unmanned air traffic management) has been established by NASA and FAA [40]. EASA is fostering UTM for European applications under the name U-space [41, 42]. Safe and secure procedures and interfaces are to be defined for incorporating a high degree of automation in air traffic management. Selected U-space services will be tailored in HorizonUAM for the application of UAM.

More details on safety and security considerations for UAM are to be published in [43].

\section{Vertidrome}

How is it possible to establish UAM ground infrastructure as safe and as reliable as possible within urban areas? What do we have to consider and what are the challenges we have to face regarding the development of an individual infrastructure component compared to an entire network? These are questions driving the HorizonUAM research regarding vertidromes.

Within the three years of duration, the objective of the HorizonUAM vertidrome research will consider the vertiport design, the UAM network management, the operation of a vertidrome network, the integration of vertiports in existent airport infrastructure and the development of the first German UAM model city to demonstrate field trials. The topics will be elaborated from a flight guidance and air transportation systems point of view. This will include the elaboration of a vertidrome's design and approach and departure procedures. The integration of UAM ground infrastructure into an airport's environment will be part of this research as well as the development of vertidrome network management approaches in order to guide and manage the vehicle flow within a vertidrome network. Furthermore, the vertidrome network will be evaluated from an operator's point of view considering the dispatching of the vehicle fleet and the allocation of resources.

The subsequent sections display the current vertidrome research status within HorizonUAM.

The work on vertidromes started by conducting an extensive literature review in order to identify the state of the art of UAM ground infrastructure with respect to the current development processes regarding vertidromes; their designs, the regulatory framework which may come into question, procedures for approach and departure intends, and the overall question of what does "on-demand" really mean. During the literature review a considerable inconsistency was found in the naming of such UAM ground infrastructure. This is the reason why we elaborated a consolidated structure within the project HorizonUAM focusing on VTOL operations (see Fig. 4). The term vertidrome is introduced here. It is used throughout the project as umbrella term for VTOL capable ground infrastructure of various sizes and with varying services to be offered.

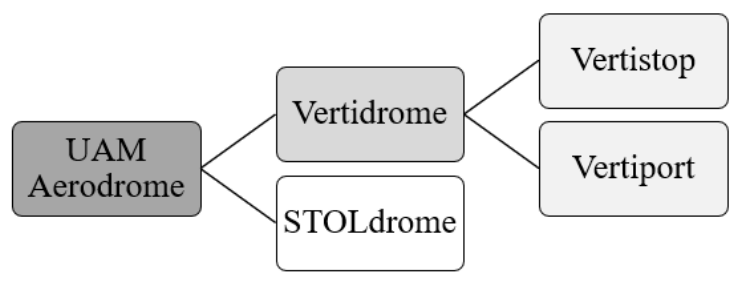

Technical Equipment (weather, communication, navigation, etc.) required for a safe operation

\begin{tabular}{|c|}
\hline UAM Aerodrome \\
\hline $\begin{array}{l}\text { - Capable of accomodating } \\
\text { every type of UAM } \\
\text { operation }\end{array}$ \\
\hline Vertistop \\
\hline $\begin{array}{l}\text { - Footprint: Small } \\
\text { - Technical Equipment } \\
\text { - No Charging } \\
\text { - No MRO Service }\end{array}$ \\
\hline
\end{tabular}

"+" symbolizes additional services (vehicle/ passenger/ staffrelated, etc.)

Fig. 4 UAM ground infrastructure: terminology used within HorizonUAM [44].

Often, already published vertidrome design concepts are focusing on showing a vision rather than providing a feasible operating concept. Driven by this insight, we developed a vertidrome concept of operation for a Linear 
Independent Expandable Drive-Through (LIEDT) topology. We proposed exemplary approaches for the layout dimensions, final arrival and departure procedures and essential elements of operational rules [44].

However, the collected vertidrome designs offer a wide range of ideas how to integrate UAM into urban and suburban environments, how to use already existent infrastructure, how to build a vertidrome "sustainable" and roughly how UAM operations can be processed. Triggered by the different vertidrome design and operation approaches, we developed a rating method - Vertidrome Airside Level of Service (VALoS) - which allows us to assess the vertidrome airside operation based on the expectations and requirements made by different stakeholders [45].

As soon as the vertidrome flight guidance strategies for sequencing of starting and landing vehicles have been finalized, the work on the optimal operation of a vertidrome network will aim to investigate vertidrome network design from an operator's perspective. Multiple network designs that vary in their degree of flexibility regarding available flight connections shall be investigated in order to enable efficient operations with regard to energy consumption, travel time and monetary costs. Therefore, a numeric traffic simulation is carried out, with agents representing the vehicles, to derive algorithms that allow an economic allocation of vehicles to specific transportation requests within that network. These methods are subsequently utilized for an assessment of UAM route networks, that are varied with regard to the number of vertidromes and the individual dispatching capacities. Finally, for a set of assumed demand patterns, the most efficient network designs are derived for different optimization metrics.

Furthermore, the advantages and disadvantages of different network design approaches are to be investigated. More precisely, slot- based and trajectory-based simulations for network designs are considered for German cities. On an international level, a collaboration agreement with NASA is in place which also addresses UAM network designs. The slot-based approach focuses on available time slots for critical infrastructure elements in a designed route network, like a vertidrome's final approach and take-off area. This design is mainly based on congestion control and a resilient route construction. On the other hand, the trajectory-based method is managing the entirety of 4D trajectories with a strategic (pre-flight) conflict detection and resolution as well as the resulting flight planning.

As a first step, a common understanding of metrics to assess the novel UAM network designs has been undertaken. This resulted in a broad overview of possible network, aerodrome, safety and human-centered and environmentally specific metrics that partially still have to be defined unambiguously. The next step is elaborating the common scenarios and use cases in detail that deal as bases for validation for the network management simulations.

In the course of the task, a simulation study on the efficiency of UAM was conducted by comparing air taxis with traditional taxicabs [46].

To include air taxi operations into the airport environment, two aspects have to be considered in particular. First, the level of safety has to be maintained. Therefore, minimum separations between conventional and air taxi traffic has to be guaranteed at all times. In addition, the risk of collisions with uncooperative airspace users such as drones or birds has to be minimized. This is especially relevant for air taxis, since they are most vulnerable to damage. Second, the operational procedures have to be adjusted to ensure an efficient handling of all air movements. To support the responsible air traffic controllers, an existing advisory system for the prevention of bird strikes is adjusted to the context of air taxi operations. Based on planned and current trajectories of aircraft and air taxis as well as on real-time movement data of drones and birds, collision risks are continuously calculated. Resulting warnings are displayed to the controller.

In this context, the airport environment to consider as well as the concepts of operations are developed in simulation. Moreover, the supporting tool for the controller is designed. These steps build the foundation for real-time human-in-the loop simulations with controllers to evaluate the operational feasibility of integrating air taxi traffic into current airport operations.

\section{Acceptance}

Any research approach aiming at investigating UAM holistically needs to consider not only technological demands on vehicles, infrastructure, and operation services, but also potential influences of human factors. In the end, the success of developing and using unmanned aerial vehicles for different purposes in urban air space will decisively depend on the degree of social acceptance of UAM in general as well as on how different UAM concepts are received by potential users and passers-by (see also [47]). On this basis, DLR will examine social acceptance by means of the following four research approaches.

First, we will conduct a large-scale telephone survey about the acceptance of civil drones and air taxis in Germany. This large-scale study will update and extend DLR's survey from 2018 [48] and aims at gaining current insights into the public's viewpoints on this rapidly developing technology. In order to consider latest trends in the research area as well as to consider focus areas of the scientific community beyond DLR, a virtual workshop with colleagues working on this issue in Germany was held in December 2020. Second, the topics participation and sustainability will 
be covered by the development and testing of an app for smartphones facilitating objective measurements and subjective assessments of noise by residents. The data of this app are going to be usable for planning and adapting flight routes and profiles such that UAM noise can be distributed as fair as possible among residents (for a detailed description of the underlying concept, see [49]; see also [50, 51]).

Third, future urban scenarios will be made tangible by means of two virtual reality (VR) studies. In one study, a UAM cabin simulator will be combined with a mixed-reality visual system and a motion platform to examine wellbeing, interaction, and comfort for passengers. In a second study, flying unmanned aerial vehicles as well as air taxis will be integrated in a $360^{\circ}$ video of an urban scene from Braunschweig, Germany, which will be presented to participants in virtual reality from the pedestrians' point of view (for a similar approach, see [52]). While the scenarios for the UAM cabin simulator study are currently being designed, the virtual reality study to simulate UAM from the perspective of passers-by is almost ready for being conducted in summer 2021. For the study last mentioned, the simulation of a prior pilot study has been expanded, especially by means of vehicle sound recordings and a specific $360^{\circ}$ video recording. One important question addressed in this study relates to the acceptance of different vehicle flight levels. Fourth and finally, accompanying research with respect to acceptance and human factors will be carried out at the request of the project partners, for example for the purpose of evaluating specific concepts developed in the current project by means of online surveys.

\section{Demonstration and Assessment}

The global assessment of the opportunities and risks of UAM covers a wide range of criteria such as impact on the urban transport system, societal benefit, environmental impact and commercial feasibility. Especially by combining the results from vehicle design, traffic forecast, and agent-based operation-simulation, total and specific energy consumption will be determined. Based on generic models, rough estimates of noise levels will be made in order to determine aircraft noise pollution and the proportion of the population affected, which is particularly relevant for public acceptance. Opportunities and risks of UAM with regard to safety and security relevant issues are also evaluated, especially with regard to official approvals.

Beyond the theoretical assessment DLR will use a variety of different simulators for the analysis and demonstration of selected project results. For example, the above mentioned UAM cabin simulator being built up for the investigation of passenger interaction and acceptance issues. The integration of UAM traffic in existing airport structures will be evaluated in an apron and tower simulator offering a $360^{\circ}$ scenery view for the air traffic controllers [53, 54].

A further aspect is the demonstration of flight scenarios within a suitable test environment to confirm theoretical assumptions of simulations with the results of trials. The demonstration flights will be conducted under usage of normed vertidromes located in a scaled test environment including a model city representing different (urban) environments (see Fig. 5 and Fig. 6). Scaled UAS (unmanned aerial systems) will be used for evaluating communication, navigation and flight guidance concepts for UAM. The advantage of test flights in a scaled test environment is the opportunity to proof theoretical assumptions made for simulation models. The results can be used to further optimize simulation parameters and to estimate the impact of UAM on different fields like expected noise pollution or traffic density.

In order to implement and demonstrate the aforementioned use cases, a scaled model city concept was designed to perform safe flight testing in an urban environment and the surroundings of the vertidromes therein. Based on a literature research [55-57] about the typical structure of European cities three building classes were defined: singlefamily houses, small apartment buildings and residential complex with three variations of height. Since, the positioning and density of buildings is determined by the urban region depending on the distance to the city center the positioning was classified in three types: detached buildings, row development, and traditional block perimeter with courtyard. A scale of 1:4 was chosen to enable also flight missions between buildings along streets having a representative width. Vertistops were integrated as flat platforms with a marking to enable their optical detection. One of the platforms is surrounded by buildings and a second one located close to residential buildings. Observation possibilities close to the vertistops are planned for acceptance studies. The designed city has a modular structure i.e. also different city structures can be realized on demand and landing sites can be temporarily relocated to e.g. higher buildings. The actual realization of the model city is planned at the National Experimental Test Center for Unmanned Aircraft Systems in Cochstedt, Germany which itself is based at an airport in operation. 


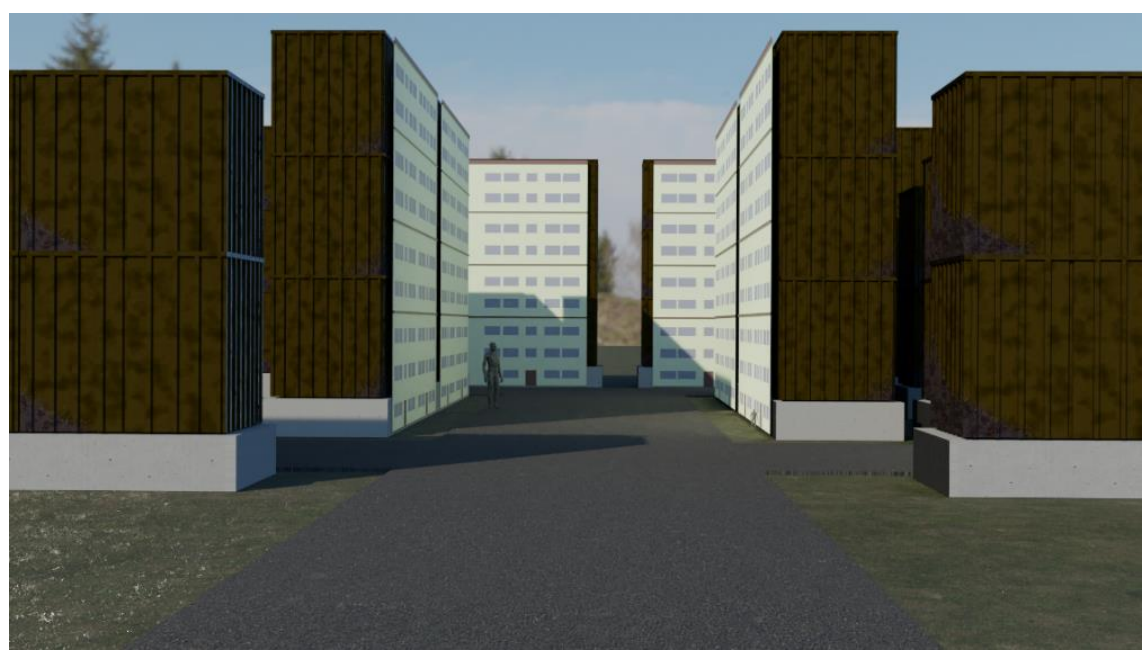

Fig. 5 Model city concept: crossroad view.

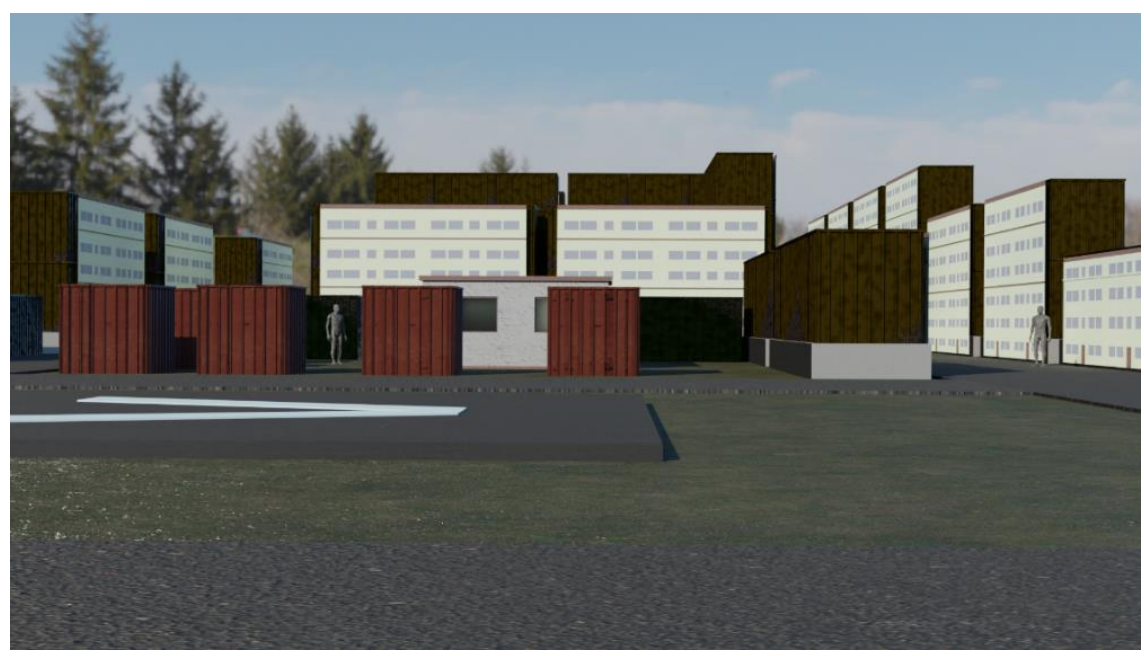

Fig. 6 Model city concept: vertistop view.

\section{Conclusion}

The DLR project HorizonUAM, running from mid-2020 to mid-2023, combines the competencies of ten different DLR institutes for a collaborative approach towards urban air mobility (UAM) research. The project deals with the research areas of UAM vehicles, infrastructure, operation, safety, security, as well as acceptance.

The paper has given an overview on the project as well as a summary of the early progress. The air taxis use cases Intra-City, Suburban Commuter, Airport Shuttle, Inter-City, and Mega-City have been defined for further assessment throughout the project. Regarding vehicle design, the focus lies on vehicles with VTOL capabilities to be used in dense urban environments. Agent-based simulations as well as a collaborative system-of-system simulation will be established to assess chances and risks of implementing UAM in German cities. Safety and security aspects are to be considered, including safe autonomy and the application of U-space services for UAM. The design and operation of vertidromes is a completely new research topic. We propose a rating method for the evaluation of the "Vertidrome Airside Level of Service".

First virtual reality studies for the investigation of human factors in UAM are about to begin. Further studies including a UAM cabin simulator, a tower simulator and field trials with scaled unmanned vehicles in a model city are to follow within the project's runtime. Further publications of the detailed HorizonUAM research results are planned. Results will also be made available to the public through annual HorizonUAM symposia ${ }^{22}$.

\footnotetext{
${ }^{22}$ https://dlr.expert/horizonuam2021
} 


\section{References}

[1] Straubinger, A., Michelmann, J., Biehle, T. „Business model options for passenger urban air mobility,“ CEAS Aeronautical Journal, No. 12, pp 361-380, 2021, doi: 10.1007/s13272-021-00514-w.

[2] Vertical Flight Society, "eVTOL Aircraft Directory,“ Electric VTOL News by the Vertical Flight Society, 2021. https://evtol.news/aircraft [retrieved 28 May 2021].

[3] FAA, "Urban Air Mobility (UAM) Concept of Operations," v1.0, NextGEN, tech. report, 2020.

[4] NASA, "UAM Vision Concept of Operations (ConOps) UAM Maturity Level (UML) 4, NASA/Deloitte Consulting LLP; 2021.

[5] Uber Elevate, "Fast-Forwarding to a Future of On-Demand Urban Transportation," Tech. rep., 2016.

[6] Volocopter, "The Roadmap tp Scalable Urban Air Mobility," white paper 2.0, 2021.

[7] EHang, "The Future of Transportation: White Paper on Urban Air Mobility Systems," 2020.

[8] Balakrishnan, K., Polastre, J., Mooberry, J., Golding, R., Sachs, P., "Blueprint for the Sky: The Roadmap for the Safe Integration of Autonomous Aircraft," Airbus Blueprint, 2018.

[9] Boeing, "Flight Path for the Future of Mobility," white paper, Boeing Next, 2019.

[10] Schuchardt, B.I., "Workload Reduction Through Steering Wheel Control for Rotorcraft," CEAS Aeronautical Journal, 10, pp. 893-902, 2019, doi: 10.1007/s13272-018-00360-3.

[11] Nieuwenhuizen, F.M., Jump, M., Perfect, P., Padfield, G., Floreano, D., Schill, F., Zufferey, J.-C., Fua, P,. Bouabdallah, S., Siegwart, R., Meyer, S., Schippl, J., Decker, M., Gusky, B. I., Höfinger M., Bülthoff H. H., "myCopter - Enabling Technologies for Personal Aerial Transportation Systems,“ 3rd International HELI World Conference, Frankfurt/Main, Germany, 2011.

[12] Publications Office of the European Union, "Metropolis, Urban Airspace Design," 2016, https://cordis.europa.eu/project/id/341508 [retrieved 28 May 2021].

[13] Sunil, E., Hoekstra, J., Ellerbroek, J., Bussink, F., Nieuwenhuisen, D., Vidosavljevic A., Kern S., "Metropolis: Relating Airspace Structure and Capacity for Extreme Traffic Densities," 11 th USA/EUROPE Air Traffic Management R\&D Seminar, FAA \& Eurocontrol, Lissabon, Portugal, 2015.

[14] Kuenz, A., "City-ATM - Live Drone Trials with Dynamic Geo-fencing," 39th DASC Digital Avionics Systems Conference, virtual, 2020.

[15] Ko, S., „High-performance lightweight construction for an innovative rescue system of the Silent Air Taxi“, Innovation report, DLR Institute of Composite Structures and Adaptive Systems, 2020.

[16] Dauer, J., Lorenz, S., Dittrich, J., "Automated Low Altitude Air Delivery," Deutscher Luft- und Raumfahrtkongress, Braunschweig, Germany, 2016.

[17] Seely, B.A., "Regional Sky Transit," $15^{\text {th }}$ AIAA Aviation Technology, Integration, and Operations Conference, 22-26 June 2015, Dallas, TX, doi: 10.2514/6.2015-3184.

[18] Crown Consulting Inc., Ascension Global, Georgia Tech Aerospace Systems Design Laboratory, and McKinsey \& Company, "Urban Air Mobility (UAM) Market Study," Tech. rep., 2018.

[19] Booz Allen Hamilton, "UAM Market Study - Technical Out Brief,” Tech. rep., 2018.

[20] Duwe, D., Keicher, L., Ruess, P., and Klausmann, F., "Quo vadis 3D Mobility," 2019, URL: http://publica.fraunhofer.de/dokumente/N-569614.html [retrieved 19 April 2021].

[21] Straubinger, A., Rothfeld, R., Shamiyeh, M., Büchter, K.-D., Kaiser, J., Plötner, K.O., „An overview of current research and developments in urban air mobility - Setting the scene for UAM introduction, " Elsevier Journal of Air Transport Management, No. 87, 2020, doi: 10.1016/j.jairtraman.2020.101852.

[22] Asmer, L., Pak, H., Prakasha, P.S., Schuchardt, B.I, Weiand, P., Meller, F., Torens, C., Becker, D., Zhu, C., Schweiger, K., and Volkert, A., "Urban Air Mobility Use Cases, Missions and Technology Scenarios for the HorizonUAM Project," to be presented at AIAA AVIATION Forum, AIAA, virtual, 2021.

[23] Volocopter, "Pioneering the Urban Air Taxi Revolution", 2019.

[24] Seider, D., Fischer, P. M., Litz, M., Schreiber, A., Gerndt, A, "Open Source Software Framework for Applications in Aeronautics and Space,". Proceedings of the IEEE Aerospace Conference, Big Sky, MT, USA, 2012.

[25] Niklaß, M., Dzikus, N., Swaid, M., Berling, J., Lührs, B., Lau, A., Terekhov, I. van, and Gollnick, V., “A Collaborative Approach for an Integrated Modeling of Urban Air Transportation System," Aerospace, 7 (5), 2020, pp. 1-34. Multidisciplinary Digital Publishing Institute (MDPI), ISSN 2226-4310, doi: 10.3390/aerospace7050050.

[26] Ciampa, P.D., Prakasha, P.S., Torrigiani, F., Walther, J.-N., Lefebvre, T., Bartoli, N., Timmermans, H., Della Vecchia, P., Stingo, L., Rajpal, D., van Gent, I., La Rocca, G., Fioriti, M., Cerino, G., Maierl, R., Charbonnier, D., Jungo, A., Aigner, B., and Anisimov, A., "Streamlining Cross-Organizational Aircraft Development: Results from the AGILE Project," Proceedings of the AIAA Aviation Forum, Dallas, TX, June 17-21, 2019, doi: 10.2514/6.2019-3454.

[27] Roland Berger, "Focus: Urban Air Mobility," Tech. rep., 2020.

[28] Porsche Consulting, "The Future of Vertical Mobility,” Tech. rep., 2018.

[29] Mayor, T., and Anderson, J., "Getting mobility off the ground," KPMG, Tech. rep., 2019.

[30] Horvath \& Partners, “Urban Air Mobility Study Report 2019: Business Between Sky and Earth,” Tech. rep., 2019.

[31] Mayakonda, M., Justin, C.Y., Anand, A. Weit, C.J., Wen, J. Zaidi, T., and Mavris, D., "A Top-Down Methodology for Global Urban Air Mobility Demand Estimation," AIAA Aviation Forum, AIAA, virtual, 2020, doi: 10.2514/6.2020-3255.

[32] Prakasha, P.S., Bertram, O., Ratei, P., Naeem, N., Nagel, B. "System of Systems Simulation driven Urban Air Mobility Vehicle Design," to be presented at AIAA AVIATION Forum, AIAA, virtual, 2021. 
[33] Prakasha, P.S., Papantoni, V., Ratei, P., Naeem, N., Nagel, B., "Urban Air Mobility Vehicle and Fleet-level Life-Cycle Assessment Using a System-of-Systems Approach," to be presented at AIAA AVIATION Forum, AIAA, virtual, 2021.

[34] Prakasha, P.S., Boggero, L., Fioriti, M., Aigner, B., Ciampa, P.D., Anisimov, K., Isianov, A., "Collaborative system of systems multidisciplinary design optimization for civil aircraft: AGILE EU project," 18th AIAA/ISSMO Multidisciplinary Analysis and Optimization Conference, p. 4142, 2017.

[35] Fioriti, M, Boggero, L., Corpino, S., Prakasha, P.S., Ciampa, P.D., Nagel, B. "The effect of sub-systems design parameters on preliminary aircraft design in a multidisciplinary design environment," Transportation Research Procedia 29: 135-145, 2018.

[36] Stolz, M., Moerland, I., Reimer, F., Hardie, T., "A User Centered Cabin Design Approach to Investigate Peoples Preferences on the Interior Design of Future Air Taxis," to be presented at DASC Digital Avionics Systems Conference, San Antonio, Texas, USA, 2021.

[37] Moerland, I., Reimer, F., Stolz, M., Bock, T.-M., "Urban Mobility: Airtaxi Cabin from a Passengers Point of View," to be presented at Comfort Congress, Chartered Insitute of Ergonomics \& Human Factors, Nottingham, UK, 2021.

[38] Schopferer, S., Donkels, A., Torens, C., Benders, S., Schirmer, S., Funke, A., Dauer, J.C., "Machine Learning Applications in Unmanned Aviation: Operational Risks and Certification Considerations", Machine Learning in Certified Systems - DEEL Workshop, virtual, 2021.

[39] Becker, D., Fiebig, U.-C., Schalk, L.M., „Approach for Localizing Scatterers in Urban Drone-to-Drone Propagation Environments," EuCAP European Conference on Antennas and Propagation, 2021.

[40] FAA, „UTM Concept of Operation, 2nd Version, “Federal Aviation Administration NextGen Office, Washington D.C., USA, 2020.

[41] EASA, „High-level regulatory framework for the U-space, Opinion 01/2020,“EASA, 2020.

[42] Lieb, J., Volkert, A., "Unmanned Aircraft Systems Traffic Management: A comparison on the FAA UTM and the European CORUS ConOps based on U-space," 9th AIAA/IEEE Digital Avionics Systems Conference, DASC, virtual, 2020.

[43] Torens, C., Volkert, A., Becker, D., Gerbeth, D., Schalk, L., Garcia Crespillo, O., Zhu, C., Stelkens-Kobsch, T.H., Gehrke, T., Daier, J.C., "HorizonUAM: Safety and Security Considerations for Urban Air Mobility," to be presented at AIAA AVIATION Forum, AIAA, virtual, 2021.

[44] Schweiger, K., Knabe, F., and Korn, B., "UAM Vertidrome Airside Operation: What needs to be considered?," Delft International Conference on Urban Air-Mobility (DICUAM), virtual, 2021.

[45] Schweiger, K., Knabe, F., and Korn, B., "Urban Air Mobility: Vertidrome Airside Level of Service Concept," to be presented at AIAA Aviation Forum, AIAA, virtual, 2021.

[46] Naser, F., Peinecke, N., and Schuchardt, B.I. "Air Taxis vs. Taxicabs: A Simulation Study on the Efficiency of UAM," to be presented at AIAA Aviation Forum, AIAA, virtual, 2021.

[47] Planing, P., and Pinar, Y., "Acceptance of air taxis - A field study during the first flight of an air taxi in a European city," 2019, https://doi.org/10.31219/osf.io/rqgpc [retrieved 3 March 03 2020].

[48] Eißfeldt, H., Vogelpohl, V., Stolz, M., Papenfuß, A., Biella, M., Belz, J., and Kügler, D., "The acceptance of civil drones in Germany," CEAS Aeronautical Journal, 11, pp. 665-676, 2020, doi:10.1007/s13272-020-00447-w.

[49] Eißfeldt, H., "Sustainable Urban Air Mobility Supported with Participatory Noise Sensing", Sustainability, 12, 3320, 2020, doi:10.3390/su12083320.

[50] Picaut, J., Fortin, N., Bocher, E., Petit, G., Aumond, P., and Guillaume, G., "An open-science crowdsourcing approach for producing community noise maps using smartphones," Building and Environment, 148, pp. 20-33, 2019, doi:10.1016/j.buildenv.2018.10.049.

[51] Guillaume, G., Can, A., Petit, G., Fortin, N., Palominos, S., Gauvreau, B., Bocher, E., and Picaut, J., "Noise mapping based on participative measurements", Noise Mapping, 3, pp. 140-156, 2016, doi:10.1515/noise-2016-0011.

[52] Aalmoes, R., and Sieben, N., "Noise and visual perception of Urban Air Mobility vehicles," Delft International Conference on Urban Air Mobility (DICUAM), virtual, 2021.

[53] Kaltenhäuser, S., "Tower and Airport Simulation: Flexibility as a Premise for Successful Research," Simulation Modelling Practice and Theory, 11 (3-4), pp. 187-196, 2003.

[54] Schier, S., Kaltenhäuser, S., „Validierungsumgebung Vorfeld- und Flugplatzkontrolle: State of the Art und Blick in die Zukunft," Deutscher Luft- und Raumfahrtkongress, Augsburg, Germany, 2014.

[55] Tröger, E., Dichte Atmosphäre: Über die bauliche Dichte und ihre Bedingungen in der mitteleuropäischen Stadt, Birkhäuser Verlag GmbH, Basel, 2015.

[56] Frerichs, S., Küpper, C., Noky, B., Simon, A., Adrian, L., Bunzel, A., Pätzold, P., Rakel, M., "Umwelt- und Aufenthaltsqualität in kompakt-urbanen und nutzungsgemischten Stadtstrukturen," Umweltbundesamt, 2018, https://www.umweltbundesamt.de/sites/default/files/medien/1410/publikationen/2018-01-29_texte_062018_stadtstrukturen.pdf [retrieved 30 March 2021].

[57] Fachverband Fußverkehr Deutschland, "Planungsprinzipien und Verkehrsflächen-Aufteilung," FUSS e.V. https://www.gehrecht.de/planungsprinzipien\#Breite-Gehwege-Regulaer [retrieved 10 October 2020]. 